\title{
Exploring and Mapping the World of Astrocytes
}

\author{
Gerald A. Dienel
}

Received: 3 July 2012/Revised: 10 July 2012 / Accepted: 13 July 2012/Published online: 4 August 2012

(C) Springer Science+Business Media, LLC 2012

Modern-day explorers use a variety of unique tools and equipment to climb unknown peaks and reveal new vistas to others. First ascent routes are named by the climbers and are described in the mountaineering and rock climbing journals, documenting the way for others to follow. Wilderness travel and neuroscience research attract pioneers like Leif Hertz, who enjoys the remote forests and mountains, as well as the unexplored areas of astrocytes, their physiology, biochemistry, signaling, energetics, neurotransmitter-related roles, and biological functions.

Leif began his scientific career in the early 1960s with Professor Mogens Schou in Aarhus, Denmark, studying the influence of monovalent cations on respiration in brain slices. He quickly followed up this initial work with assays of the effects of $\mathrm{K}^{+}$and $\mathrm{Na}^{+}$on respiration in astrocytes acutely-dissected from adult brain under the auspices of Erik Zeuthen at the Carlsberg Laboratory, Copenhagen. Professor Holger Hydén, Göteborg, Sweden, was Leif's spiritual mentor who provided inspiration and perspective in his early years. Most of Leif's subsequent studies utilized primary cultures of astrocytes as a model system to evaluate properties of astrocytes. Astrocyte cultures were developed by Monique Sensenbrenner in Paul Mandel's laboratory in Strasbourg, and collaborations between Leif and his wife Elna, who prepared almost all of the cultures for many years, led to many co-authored publications. An important underlying theme

Special Issue: In Honor of Dr. Leif Hertz.

G. A. Dienel ( $ه)$

Department of Neurology, University of Arkansas for Medical

Sciences, Shorey Bldg. Room 715, Mail slot 830,

4301 W. Markham St, Little Rock, AR 72205, USA

e-mail: gadienel@uams.edu

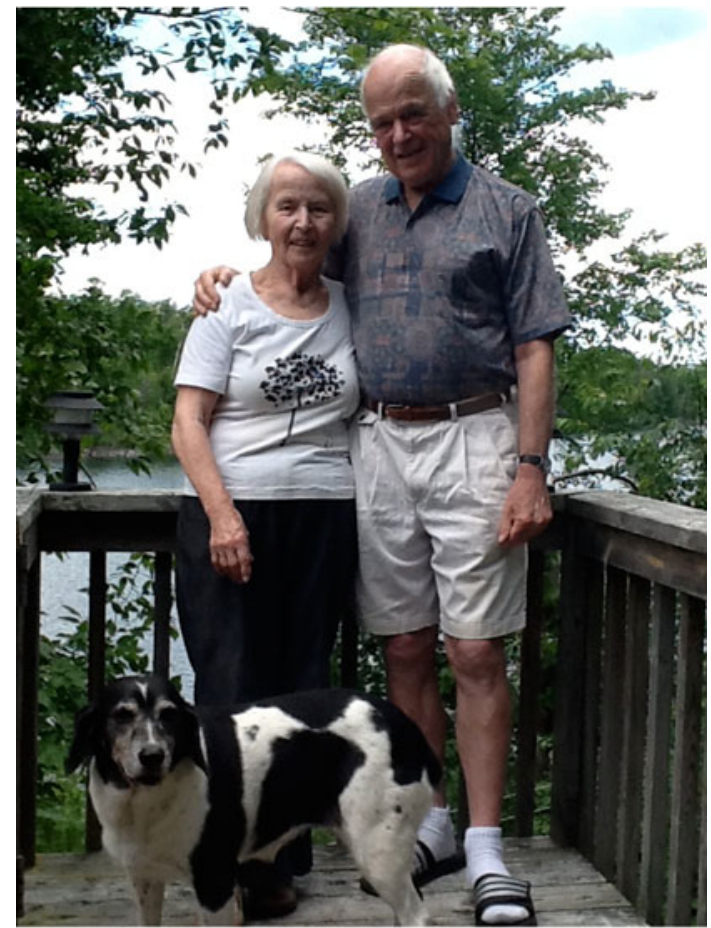

Dr. Leif Hertz, Elna Hertz, and Spot

of Leif's work throughout more than 50 years of research has been interactions between $\mathrm{K}^{+}$uptake and energy metabolism in astrocytes, i.e., interfacing with neuronal activity. From this center, Leif's interests have led in many directions, including the roles of astrocytes in glutamate, glutamine, and GABA metabolism (with Arne Schousboe) and the interactions of energy metabolism with neurotransmitter turnover and receptor activity in astrocytes. Leif's work involved students, fellows, and many collaborating scientists, and his laboratory was supported for more than 20 years by the Medical 
Research Council in Canada after his move to Canada in 1974. This MRC support made possible many novel studies on cultured astrocytes that helped pave the way for design and evaluation of in vivo studies on astrocytes carried out by others using stable isotopes and magnetic resonance spectroscopy. Indeed, $\left[{ }^{13} \mathrm{C}\right]$ glucose and $\left[{ }^{13} \mathrm{C}\right]$ acetate studies have demonstrated high rates of glucose oxidation in astrocytes in living human and animal brain, consistent with results of the earlier experiments carried out by Leif, Elna, and their colleagues in differentiated, cultured astrocytes from rodent brain. After retirement, Leif has had the good fortune to continue to participate in studies with the research team headed by Liang Peng, his previous Ph.D. student and now professor at China Medical University, Shenyang, China. Leif's work was previously recognized with the publication of a special issue of Neurochemistry International in his honor (vol. 36, number 4-5, 267-482, April, 2000). This issue includes a "Certificate of Recognition for his pioneering role in developing techniques for the primary culture of neuronal and glial cells from brain, and for his studies which have contributed greatly to the understanding of metabolism in astrocytes and neurons and neuronal/glial interactions in the brain." This award was presented to Leif in 1997 by his Neurochemistry colleagues at the Third International Conference on Brain Energy Metabolism in Waterville Valley, NH. Roger Butterworth and Arne Schousboe, Guest Editors of the first special issue entitled, "Astrocyte Function", provided an overview of Leif's academic career and achievements, so this information is not included here. Because the material covered by the articles in the Neurochemistry International special issue is still relevant today, the emphasis of the present special issue involves different topics and authors.

The Neurochemical Research special issue, entitled "Current Astrocyte Research", has six overall themes related to Leif's major, life-long interests. These topics bring a variety of attributes of astrocytes to the forefront: (1) complex physical and dynamic structures of astrocytes, (2) model systems using astrocytes, (3) $\mathrm{K}^{+}$clearance and water movements, (4) metabolic specialization and metabolite trafficking, (5) signaling and cell-cell communication among astrocytes and neurons, and (6) astrocyte functions in pathological conditions. Together, the two special issues cover a broad spectrum of investigations into the properties and functions of astrocytes, and they serve as important resources.

Astrocyte biology is not without controversy, and some of the current 'hot topics' are not addressed in this special issue because they have been recently reviewed elsewhere. Apparently-discrepant results are important because they can drive new experiments to evaluate the basis for data that cannot be explained with current knowledge.
Discordant results obtained by different, established laboratories necessarily bring attention to the properties of astrocyte preparations that are governed, in part, by culture conditions. Gene expression and phenotype are influenced by culture medium constituents (including micronutrients, glucose concentration, potential growth factors, and signaling compounds), extent and duration of differentiation, time in culture, and the presence or absence of other cell types that can release cytokines and other regulatory compounds. Special mention must be made of the use of high-glucose media (10-50 mmol/L) that are used by many researchers for culture of astrocytes and neurons; chronic hyperglycemia carries the risk of adverse consequences. Leif and colleagues routinely use low-glucose media for astrocyte cultures (neuronal cultures cannot tolerate medium changes, and higher levels of glucose are commonly used), and supplement the medium with dBcAMP to induce morphological and developmental changes, L-channels for $\mathrm{Ca}^{++}$, and other responses. The extent of functional development in culture is critical to interpretation of results, and Leif and his collaborators have always made strong efforts to define the properties of their cultures and compare them to those of astrocytes in living brain. The late-term development of glutamine synthetase in cultured astrocytes indicates that short-term cultures do not possess at least one of the hallmark attributes of astrocytes, i.e., the ability to convert ammonia plus glutamate to glutamine and to participate fully in the glutamate-glutamine cycle between astrocytes and neurons. Expression of one or two astrocyte-specific markers in young cultures does not mean that they represent the 'normal' astrocyte in living brain. Indeed, astrocytic maturation in brain in vivo involves long-term, region-specific interactions with neurons and other cell types. Thus, observations in culture need continuous interactions and interplay with data obtained in vivo. Leif's work showing that astrocytic uptake of glutamate is more than capable of metabolically supporting its own uptake is consistent with results of other laboratories and in vivo studies that reveal high oxidative activity of astrocytes. Astrocytes are versatile cells that increase fluxes in their glycolytic and oxidative pathways to carry out established and unidentified ATP-dependent functions during brain activation. Leif's work has helped elucidate these functional characteristics of astrocytes, and stimulate in vivo studies of astrocytic activity.

Critical interpretation of data is the hallmark of science, and the transition from a working in vitro model to an accepted portrayal of cellular interactions in living brain is a very complex process requiring integration of quantitative in vivo studies into the model system. Observations in cultured astrocytes that are not consistent with results obtained in vivo cannot be correct unless the discrepancy can be explained. Metabolic activity, capacity, and 
development, metabolic responses to stimulatory conditions, and metabolic support of astrocytic and brain functions are areas in which Leif Hertz and colleagues have made important contributions. Astrocytes contribute significantly to overall brain energetics and function, and these attributes need to be taken into account when interpreting metabolic brain imaging studies.

\section{A Signal to Researchers from a Glycolytic-Oxidative Complex: The Astrocyte}

It all starts with a stimulus and discharge

Ionic imbalance and recovery

Transmitter release and recharge

In the shadow of neurons

Astrocytes bloom

When signals abound

Purified cultures tell some of their story

They provide directions, capabilities, and mechanisms

They give directions for more complex assays

In complicated environments

Using expensive tools

Do the signals that we image during activation

Come from astrocytes, or neurons, or both?

Excitatory transmission triggers many downstream events

Which of these provides the signals

For our detectors

Can we design the right experiment

Or do we accept incomplete results

Where is the lactate shuttled

Neuroscience is so befuddled

Supportive data by some are cuddled

Whereas others find them muddled

A controversy persists unresolved

Until critical thinking and rigorous experimentation

Uncover the missing links

Hidden among astrocytic activities

Perhaps cation fluxes, syncytial trafficking,

Receptor-mediated signaling, gliotransmitter activity,

Oxidative metabolism, and compartmentation

We anticipate the reports of explorers and pioneers

Documenting their forays into new territory.

\section{“READ OR DIE!"}

(C) Gémino H. Abad, Ph.D.
Excerpts from Recognition Day Speech by University Professor Emeritus Gémino Henson Abad, University of the Philippines (UP), College of Arts and Letters, UP Film Institute, 16 April 2011, Reprinted with the permission of Dr. Abad.

"I have only a one-word message, and I could end right there: Read! ,"

"You see, upon graduation, your education begins, you were only equipped for it in college. The word "education" is from Latin, e-ducere, "to lead out of." Out of what? - out of any darkness of mind: out of ignorance, prejudice, fanatic advocacy.

It is a curious thing that the word "dogma" is from Greek dokein, meaning "to seem, or to seem good," which is by definition what an opinion is: dokein. An opinion (Latin, opinari, to suppose, imagine, or conjecture) is anything that hovers between fact and fiction, with more or less of either one.

It is also instructive that "theory" is from Greek theoria, meaning "a way of seeing." Thus, any theory one might subscribe to is only a way of looking, of making sense. No theory has a monopoly of seeing. And further, "to subscribe" is, again, from Latin sub-scribere, that is, "to be written under," and so, if I subscribe to any theory, I see things in light of a script that is already given. By any theory, what I see and understand is a reading of a given script by which I too am read.

From all these, you can see that I am a skeptic, again from Greek skeptesthai, that is, "to look, to consider, to ponder." That was what your college education equipped you for: a life of the mind, a care for thought that, this side of Eden, is our only light. Look and consider, "to read close is to open."

(For the complete text and more information about the author, see: http://www.allvoices.com/contributednews/8816146-read-or-dieup-cal-recognition-dayspeech-of-up-university-prof-emeritus-gemino-hensonabad-16-april-2011-up-film-institute; http://en.wikipedia. org/wiki/G\%C3\%A9mino_Abad)

Pioneers need to know the geography and history of the field, as well as the depth and breadth of relevant experiments. Young and experienced investigators alike need to read widely and understand the basis for conclusions drawn from different data sets. From this knowledge base, scientific discoveries move forward into uncharted territory. Those who are not skeptical are limited by the boundaries of dogma and theory; they become repetitious and unimaginative. Researchers need to see beyond the images 
of past thought or interpretation so they can open new doors into the world of astrocytes. Leif's life work is derived from an insatiable curiosity, a critical mind, a wealth of knowledge, detailed experimentation, and thoughtful evaluation. His work has emphasized, but has not been restricted to, metabolic and oxidative activity in astrocytes, with linkage to cation fluxes, neurotransmission, receptor signaling pathways, pharmacological actions, and memory and learning. Leif has inspired many researchers to extend the horizons of astrocytic functions. From his perspective at the pinnacle of a long, fruitful career Leif can have the joy and satisfaction of seeing confirmation, translation, and extension of his early work that revealed high respiratory rates in astrocytes and the energy-requiring roles of astrocytic $\mathrm{K}^{+}$uptake during neuronal excitation.
As guest editor, it has been my privilege to help bring this special issue to fruition. I thank all of the authors for their strong contributions to this issue and to the field of astrocyte biology. I also thank the Chief Editor of Neurochemical Research and Springer Publishers for making this special issue possible. The depth, breadth, and high quality of the articles in this issue are a tribute to the work of Leif and Elna Hertz and their students, fellows, and collaborators who have explored and mapped many important aspects of the world of astrocytes. We celebrate their accomplishments with this special issue that defines and extends some of the boundaries of current knowledge. The articles describe fascinating research, and we look forward to new discoveries. 